\title{
4 Teambesprechung und Teamentwicklung
}

\section{Christine Kuch}

Teambesprechungen werden nicht umsonst als zentrales Element eines lebendigen Qualitätsmanagements bezeichnet. Hier können Veränderungen mit allen Beteiligten besprochen und Informationen, die für alle von Belang sind, weitergegeben werden. Die Zusammenarbeit wird gestärkt und eine angenehme Teamkultur in der Praxis gefördert. Zugleich ist die Teambesprechung ein wichtiges Instrument zur Praxisführung.

Nun könnte man sich einfach daran machen und Teambesprechungen einführen, schließlich werden sie auch in den Richtlinien des Gemeinsamen Bundesausschusses gefordert. In vielen Fällen sind Teambesprechungen von Anfang an erfolgreich, wenn man sich erst einmal den Ruck gegeben hat, und den Vorsatz in die Tat umsetzt.

Für diejenigen, die sich diese Sitzung effektiver wünschen, bei denen die Besprechung nicht „rund läuft", sind in diesem Kapitel einige „Tipps und Tricks“ zusammengetragen, die auf langjähriger Erfahrung und vielerlei Diskussionen mit Lehrgangsteilnehmern und Beratungs- und Coachingkunden basieren.

Für die effektive Durchführung einer Teambesprechung sind diese nachstehend weiter ausgeführten Punkte relevant:

grundlegende Zielsetzung der Teambesprechung klären

- Termine und Rhythmus klären: Rituale helfen

- Tagesordnung - oder: Wie kommen die relevanten Themen aufs Tablett? Vorher!

- Protokoll während der Besprechung machen und zur Moderation nutzen

- Besprechung moderieren - und nicht zugleich leiten wollen! 
- Gesprächskultur: Alle zu Wort kommen lassen

- Konflikte nicht unter den Teppich kehren

- hin und wieder "Nabelschau“: Teamentwicklung braucht Raum!

\subsection{Zielsetzung der Teambesprechung klären}

Vor der Einführung von Teambesprechungen sollte man als Praxisinhaber überlegen, wozu man eine solche Besprechung einführen möchte. Um welche Themen geht es für Sie vor allem?

- Verbesserungsideen der Teammitglieder besprechen?

- Ziele kommunizieren?

- Meinungsverschiedenheiten klären?

- Mitarbeiter schulen?

- Auf Missstände hinweisen?

- Informationen zu neuen Abrechnungsmodalitäten weitergeben?

- ...?

Wenn Sie die Zielsetzung für sich geklärt haben, prüfen Sie zunächst noch einmal nach, ob eine Teamsitzung das geeignete Instrument für die Zielerreichung ist. Beispielsweise ist eine Teambesprechung kaum der Ort, einzelne Mitarbeiter effektiv zu kritisieren - der einzige Effekt wäre hierbei ein „Stimmungstief“ "im Team. Der Vorteil explizierter Ziele ist, dass man in der Folge die Teamsitzungen effizienter und effektiver auf diese Ziele hin führen kann. Um diesen Effekt noch zu unterstützen, überlegen Sie bitte, woran Sie konkret erkennen können, dass das Ziel erreicht wird:

- „Mindestens 3 Verbesserungsvorschläge der Teammitglieder wurden besprochen."

- „Konflikte zwischen den Mitgliedern gehen deutlich zurück.“

- usw.

Außerdem können Sie jeweils nach einer Besprechung prüfen:

" „Wie groß ist mein Redeanteil?“(Der Redeanteil der Ärzte sollte geringer sein als derjenige der Teammitglieder, sonst handelt es sich bei dem Treffen kaum um eine Teambesprechung.)

- „Beteiligen sich alle Teammitglieder?“

- „Ist die Atmosphäre so, dass man offen seine Meinung sagen kann?“

- usw.

Sollten Sie einmal den Eindruck haben, die Besprechung sei weniger geglückt, überlegen Sie bitte nicht, warum das der Fall war. Denken Sie lieber an eine gute Besprechung in der Vergangenheit, und überlegen Sie, was diese Besprechung so gut gemacht hat: Wie haben wir es damals geschafft, unsere Ziele zu erreichen? Wenn Sie mindestens 10 Punkte gefunden haben (Erna hat sich vorher um die Themen gekümmert, ich habe Kekse gekauft, wir ha- 
ben gelacht, Sabine hat mich unterbrochen, wenn ich wieder mal zu lange geredet habe, Janine hat einen Vorschlag gemacht und alle waren ganz gebannt, ...), überlegen Sie bitte, welche dieser Punkte Sie selbst beeinflussen können. Schließlich denken Sie darüber nach, was Sie konkret in der aktuellen Situation verändern möchten, um die nächste Teamsitzung wieder erfolgreicher zu gestalten. Nehmen Sie sich eine Sache vor, die Sie selbst leicht und mühelos umsetzen können. Meistens sind es kleine Punkte, die man im Alltagstrubel vergisst - es kommt einfach darauf an, damit (selbst!) anzufangen.

\subsection{Termine und Rhythmus klären: Rituale helfen}

Auch wenn man erst einmal den Eindruck hat, überhaupt keine Zeit für Teamsitzungen zu haben: Regelmäßige Termine sind essenziell. Denn nur dann kann ich mich als Mitarbeiterin darauf einstellen, mir im Vorfeld Gedanken machen, mich darauf verlassen, dass ich Dinge in Ruhe ansprechen kann und gehört werde. Wenn Teambesprechungen immer wieder ausfallen, sollte man „zurück auf Start“ gehen und überlegen, was denn lohnenswerte Ziele der Besprechung sind. Dann fällt es sicherlich wieder leichter, Zeit zu investieren.

Ein anderes Argument in Bezug auf die Ressource Zeit: Anderen Praxen ergeht es nicht anders, alle haben viel zu tun - und trotzdem schaffen es viele, Zeit für Teambesprechungen zu reservieren. Die übrigens zur Arbeitszeit und nicht zur Freizeit der Mitarbeiter gehört.

Teambesprechungen sollten mindestens einmal im Monat stattfinden, damit man sich als Leitung ausreichend, außerhalb der Routine und nicht zwischen Tür und Angel, um gute Arbeitsbedingungen des eigenen Teams kümmern kann. Die Besprechungen sollten nach Möglichkeit nicht länger als eine bis maximal zwei Stunden dauern, um eine effektive Arbeit zu gewährleisten. Sie sollten allerdings auch nicht zu kurz angesetzt werden: In 15 Minuten ist es eher unrealistisch, dass beispielsweise Konflikte angesprochen werden können.

\subsection{Tagesordnung - oder: Wie kommen die relevanten Themen aufs Tablett? Vorher!}

Für die effektive Durchführung der Sitzung ist es wichtig, dass am Anfang feststeht, welche Themen heute besprochen werden. Die Themen sollten schon vorher gesammelt werden, damit sich die einzelnen Teilnehmenden gedanklich vorbereiten können und nicht erst in der Sitzung anfangen zu überlegen. Zudem sollten die Themen vor allem von den Mitarbeitern vorgeschlagen werden, schließlich handelt es sich hier um ihre Besprechung. Für Möglichkeiten der Themensammlung sind der Phantasie keine Grenzen gesetzt, um hier nur einige zu nennen: 


\section{Tagesordnung Team}

14.2.2010

$\begin{array}{ll}\text { 1. Reorga Labor } & 30 \text { Minuten } \\ \text { 2. Urlaubsplan } & 2 \text { Minuten } \\ \text { 3. Einarbeitung } & 10 \text { Minuten } \\ \text { 4. EBM-Infos } & 10 \text { Minuten } \\ \text { 5. Verschiedenes } & \text { offen }\end{array}$

Abb. 11 Tagesordnung Team mit Zeitplan

- große Tafel in der Küche, an die jeder Themen schreibt

- Kiste, in die man Zettel mit Themenvorschlägen wirft

- Themenbuch, das am Tresen liegt und in das jeder Eintragungen vornimmt

- Aufgabenordner „Team“ in Outlook, auf den jeder Zugriff hat

- Patient „Dreamteam“ in Praxissoftware anlegen, der Themen verordnet bekommt

Für eine effiziente Durchführung der Besprechung ist es besonders hilfreich, zu Beginn der Sitzung kurz alle Themen durchzugehen und zu überlegen, wie viel Zeit welches Thema benötigt. Das mag zunächst etwas umständlich klingen, hilft aber enorm dabei, dass alle sich für die Zeit verantwortlich fühlen. Damit wird auch sichergestellt, dass alle Themen besprochen werden - anstelle des noch häufig üblichen Verfahrens, dass nur ein oder zwei Themen ausführlich besprochen und „die letzten zehn Punkte“ schnell „abgehakt“ werden, wenn alle schon den Besprechungsraum verlassen. Denn das führt dazu, dass niemand genau weiß, was eigentlich beschlossen wurde - diese Zeit kann man sparen. Die Tagesordnung (s. Abb. 11) sollte für alle sichtbar sein, die benötigen Zeiten werden zu Beginn in einer kurzen Absprache im Team eingetragen - und los kann es gehen.

\subsection{Protokoll während der Besprechung machen und zur Moderation nutzen}

Dass man überall den Hinweis lesen kann, ein Protokoll sei unerlässlich, wundert nicht: Schließlich passiert so viel im Alltag, dass man sich kaum daran erinnern kann, was vor einem Monat oder gar vor zwei oder drei Monaten besprochen wurde. Zumindest kann man davon ausgehen, dass verschiedene Beteiligte sich an unterschiedliche Dinge erinnern. Sinnvoll ist ein solches schriftliches Protokoll also schon, aber wo, bitteschön, soll man denn die Zeit hernehmen, auch noch ein Protokoll zu schreiben? Die Lösung lautet: Schreiben Sie das Protokoll während der Sitzung, und zwar so, dass es alle sehen 


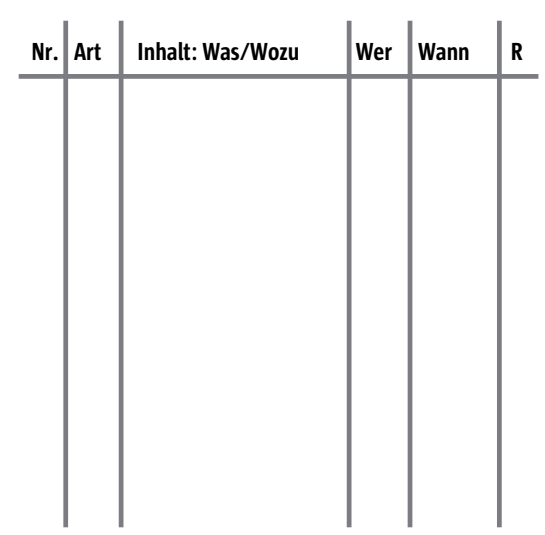

Abb. 12 Maßnahmenplan (Ergebnisprotokoll)

können. Die Investitionen von circa 6o € in eine Flipchart lohnt sich. Denn dann kann das Protokoll, das auf der Flipchart notiert wird, gleich zur Moderation genutzt werden: Diejenige, die das Protokoll an der Tafel schreibt, kann immer wieder strukturierend in das Gespräch eingreifen: „Was soll ich da jetzt genau schreiben?“, „Muss da jemand etwas tun?“, „Bis wann?“, „Wer macht was?“, „Und wann hören wir wieder davon?“ Ein sogenannter Maßnahmenplan (s. Abb. 12) bewährt sich:

Unter „Nr.“ trägt man eine fortlaufende Nummer der jeweiligen Einträge ein. Unter „Art“ wird entweder „M“ für „Maßnahme“, „E“ für „Entscheidung“ oder „I“ für „Information“ notiert, je nachdem, um was es sich hier handelt. Unter „Inhalt“ wird beschrieben was, und ggf. wozu etwas getan werden muss. Unter „Wer“ und „Wann“ werden Verantwortliche und das Datum der Erledigung notiert, unter R, wann wieder in diesem Kreis über den Punkt gesprochen wird.

\subsection{Besprechung moderieren (und nicht zugleich leiten wollen)}

Die Moderation von Teambesprechungen kann als eine der sichersten Methoden gelten, eine effiziente und effektive Durchführung zu erreichen. Bei dieser Moderation geht es, im Gegensatz zur reinen Gesprächsmoderation à la Will oder Christiansen, darum, eine Gruppe dabei zu unterstützen, ein gemeinsames Arbeitsergebnis zu erzielen. Zielsetzung der Moderation ist, eine Beteiligung aller Teilnehmenden zu erreichen, das Wissens- und Kreativitätspotenzial der gesamten Gruppe zu nutzen und alle Ideen, Meinungen und Ansichten kommentarlos zu sammeln. Die Gruppe wird dabei unterstützt, Themen ergebnisorientiert und effektiv zu bearbeiten und gemeinsame Entscheidungen zu treffen. Eine Moderatorin hat „den Hut auf“ für die methodische Gestaltung der Besprechung, gibt inhaltliche Fragen an die 
Gruppe zurück und fragt, anstatt zu behaupten. Sie hält sich mit inhaltlichen Äußerungen zurück und ergreift nicht Partei für die eine oder andere Ansicht. Die im letzten Punkt angesprochene Neutralität oder „Allparteilichkeit" macht deutlich, dass es sehr schwer ist, eine Sitzung gleichzeitig inhaltlich zu leiten und eine Gruppe zu moderieren. Wie soll man sich inhaltlich zurückhalten, wenn man dafür den Hut aufhat? Während es bei der Leitung um die Frage geht, was gesprochen wird, geht es bei der Moderation darum, wie gesprochen wird. Es empfiehlt sich sehr, die beiden Rollen zu trennen und als Leitung nicht zu moderieren. Der Erfahrung nach ist dies für viele Leitungskräfte deutlich entlastend. Zudem kann es äußerst hilfreich sein, eine medizinische Fachangestellte mit der Moderation zu beauftragen - das führt in aller Regel zu wesentlich besseren Ergebnissen. Cleichzeitig muss dann aber auch gewährleistet sein, dass der Arzt oder die Ärztin sich nicht einfach über Vorschläge des Moderators hinwegsetzen darf, sondern sich wie alle anderen Teilnehmenden in die Cruppe einfügt. In aller Regel reicht es für den Anfang aus, wenn man sich als Moderator vornimmt, sich möglichst nicht ins Gespräch inhaltlich einzumischen (bzw. wenn, dann deutlich zu machen, dass man gerade nicht „als Moderator“, sondern als Mitarbeiter spricht), darauf zu achten, dass sich alle gleichmäßig beteiligen, und schließlich noch die Zeit im Blick zu haben. Wer sich dann für eine größere Methodenvielfalt interessiert, kann sich ein Buch zu dem Thema Moderation oder Kreativitätstechniken kaufen (einfach eine Buchhandlung aufsuchen) und selbstverständlich gibt es auch die Möglichkeit, sich über die KVen oder andere Anbieter als (Qualitätszirkel-)Moderator ausbilden zu lassen.

\subsection{Gesprächskultur: Alle zu Wort kommen (lassen)}

Noch ein kurzes Wort zur Gesprächskultur: Auch der beste Moderator ist schnell überfordert, „das ganze Team einzubinden“, wenn die Regel gilt „Der Doktor hat immer Recht und darf immer dazwischenreden. "Insbesondere die an der Teambesprechung teilnehmenden Ärzte können ganz maßgeblich und positiv auf die Gesprächskultur einwirken. Das heißt ganz konkret: sich zurückhalten, Äußerung von Mitarbeitern unterstützen - auch wenn sie mal gegen die eigene Meinung gehen, Fehler eingestehen, kurz und gut: Vorbild sein!

\subsection{Konflikte nicht unter den Teppich kehren}

Dass man Konflikte nicht unter den Teppich kehren sollte, ist leicht gesagt. Den meisten Menschen fällt es recht schwer, Missstimmungen offen anzusprechen. Man befürchtet negative Reaktionen, ist sich unsicher, hat „ja auch nur so ein komisches Bauchgefühl". Dennoch darf auch an dieser Stelle 
nicht der Appell fehlen: Je früher Sie eine Missstimmung ansprechen, umso leichter kann sie aus der Welt geräumt werden. Und nicht jeder Konflikt ist negativ: häufig treffen hier unterschiedliche Perspektiven aufeinander, aus denen man lernen kann. Unglücklich wird der Konfliktverlauf erst dann, wenn man immer weiter macht, die Fronten sich verhärten und persönliche Verletzungen entstehen. Aber gerade dann geht es nicht darum, aufzuarbeiten, wie es denn zum Konflikt gekommen ist (das ist eher ein probates Mittel, um den Konflikt aufrecht zu erhalten). Statt problemorientiert zwischen verhasster Vergangenheit und befürchteter Zukunft hin- und herzuschwanken, macht man es sich leichter, wenn man schaut, was denn die gemeinsamen Ziele der Konfliktparteien sind, und wie man in kleinen Schritten dort hinkommt. Ein an Lösungen orientierter Umgang mit Konflikten verzichtet auf Ursachenforschung und Schuldzuweisungen und versucht eher danach zu schauen, welche Perspektiven sich ergeben - und wie diese, nach und nach, erreichbar sind.

\subsection{Hin und wieder „Nabelschau“: Teamentwicklung braucht Raum!}

Die Möglichkeit zur Selbstreflexion ist eine der wichtigen Kompetenzen zur Persönlichkeitsentwicklung. Ähnliches gilt auch für Gruppen: Die Beschäftigung mit sich selbst, die manch einem zunächst ein Greuel ist, zeigt positive Konsequenzen. Wo stehen wir als Team? Wie geht's uns? Was läuft gut in der Zusammenarbeit? Was könnte dabei besser laufen? - für diese oder ähnliche Fragen sollte man sich zumindest ein- bis zweimal im Jahr Zeit nehmen - dann lassen sich auch mal besonders stressige Zeiten leichter überstehen und das Team kann seine volle Leistung bringen. Ein systematisches Vorgehen für eine solche Zusammenkunft kann man dem lohnenswerten Buch von Daniel Meier (Meier 2005) entnehmen. Das Vorgehen stellt sicher, dass sowohl positive wie negative Dinge leicht angesprochen werden können und leitet ein Team dabei sehr ressourcenorientiert an, sich auf der Basis des schon Erreichten weiter zu entwickeln.

\section{Aufgabe 14 für anwendungsfreudige Leser}

Überlegen Sie bitte: Welcher der aufgeführten Punkte hat Sie in Bezug auf Ihr Team besonders angesprochen? Oder vielleicht fällt Ihnen gerade ein für Sie (bzw. Ihr Team) wichtiger Punkt ein, der hier nicht benannt wurde? Besprechen Sie diesen Punkt in Ihrem Team und überlegen Sie gemeinsam, wie Sie das, was Sie sich vornehmen, verbindlich umsetzen können.

\section{Literatur}

Meier D: Wege zur erfolgreichen Teamentwicklung. Books on Demand 2004 